Kompass

Neumología

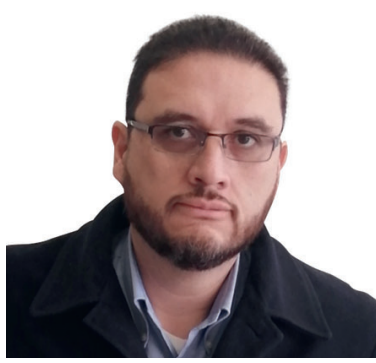

\title{
Hipertensión pulmonar en pacientes con fibrosis pulmonar idiopática: Evaluación de la terapia con antifibróticos
}

\author{
Marco Hugo Sánchez-Bustillos
}

Hospital Star Médica, Chihuahua, Chihuahua, México

Resumen de Tahara M, Oda K, Yamasaki K, et al.: Temporal echocardiographic assessment of pulmonary hypertension in idiopathic pulmonary fibrosis patients treated with nintedanib with or without oxygen therapy. BMC Pulm Med 2019;19(1):157.

\section{Keywords}

Echocardiography · Idiopathic pulmonary fibrosis · Long-term oxygen therapy · Nintedanib · Pulmonary hypertension

\footnotetext{
Abstract

Background: Nintedanib is an inhibitor of receptor tyrosine kinases, including vascular endothelial growth factor receptor, but its effects on pulmonary hypertension (PH) in idiopathic pulmonary fibrosis (IPF) patients with chronic hypoxia were unclear.

Methods: This study included a nintedanib prospective study and historical control study. In the nintedanib prospective study, pulmonary artery systolic pressure (PASP) measured using transthoracic echocardiography was evaluated at six points during 48 weeks in 16 IPF patients in whom nintedanib was started. In the historical control study, adjusted annual change in PASP was between patients treated with $(n=16)$ and without $(n=15)$ nintedanib.
}

Results: In the nintedanib prospective study, the mean PASP at 48 weeks after starting nintedanib was significantly higher compared to that at baseline. When IPF patients were divided into two groups, IPF patients with or without long-term oxygen treatment (LTOT), mean PASP at 48 weeks was significantly higher than that at baseline only in IPF patients receiving LTOT ( $P=0.001)$. In the historical control study, adjusted annual change in PASP in IPF patients treated with nintedanib was significantly lower than that in patients treated with no antifibrotic agents when considering patients without LTOT $(0.26 \mathrm{mmHg}$ vs $7.05 \mathrm{mmHg}$; $\mathrm{P}=0.011)$.

Conclusions: We found differential effects of nintedanib on $\mathrm{PH}$ between IPF patients with or without LTOT. Nintedanib may have a disadvantageous effect on PH in IPF patients with LTOT. Conversely, nintedanib treatment may be beneficial to PH in IPF patients without LTOT.

(c) 2019 The Author(s) 


\section{Transferencia en la práctica}

\section{Contexto del estudio}

La fibrosis pulmonar idiopática (FPI) es una enfermedad severa que de ser vista como una patología inflamatoria, hoy es entendida como una fibrosis proliferativa. Este estudio evalúa el efecto de Nitedanib (N), un antifibrótico inhibidor de la tirosinquinasa, y con ello varios factores de crecimiento implicados en la formación de fibrina, como el factor de crecimiento endotelial vascular (VEGF) cuya inhibición se ha relacionado con aumento de la hipertensión pulmonar (HP). Surge aquí la hipótesis que el Nitedanib al inhibir el VEGF aumente la HP.

Este estudio compara individuos expuestos a Nitedanib, contra aquellos que no lo están, evaluando en incremento de HP en cada uno de ellos.

\section{Resultados del estudio}

Existen dos brazos uno prospectivo de pacientes con FPI tratados con Nitedanib, evaluados con ecocardiograma transtorácico (ECTT) periódicamente midiendo la presión sistólica de la arteria pulmonar (PSAP). El segundo brazo fue retrospectivo en pacientes con FPI tratados sin antifibróticos, se realizó ECTT en forma anual. Este grupo hace las funciones de grupo control. En ambos grupos se eliminó a prácticamente la mitad de los pacientes que iniciaron los cuales no se incluyeron en el análisis. Ambos grupos se dividieron en los que reciben oxigenoterapia continua en domicilio (OCD) y los que no.

Se formaron 4 grupos:

- El tratado con Nitedanib que requiere $\operatorname{OCD}(N+O C D) n=9$.

- El sin tratamiento antifibrótico que requiere OCD (STAF + OCD) $\mathrm{n}=10$.

- El grupo de Nitedanib sin OCD (N) $n=7$.

- El sin tratamiento antifibrótico sin OCD (STAF) $n=5$

Los grupos se muestran heterogéneos, hay diferencias en las condiciones determinantes como lo son la disnea, la difusión de monóxido de carbono (DLCO\%), y la presencia de enfisema. En el grupo $\mathrm{N}+$ OCD el 56\% de los pacientes padece enfisema, en el N fue de $14 \%$, y en el STAF + OCD fue del 30\%. Otro sesgo es que el grupo de OCD tenga DLCO\% menor de aquellos sin OCD, 49\% en el $\mathrm{N}+$ OCD y $40 \%$ en el STAF + OCD en comparación de 70\% del grupo $\mathrm{N}$ y $74 \%$ del grupo SATF. La disnea en el $\mathrm{N}+\mathrm{OCD}$ el promedio fue de 3 del mMRC, en el STAF + OCD fue de 2 y en el $N$ fue de 1 , el primer grupo presenta peor clase funcional y se espera un deterioro más acelerado.

La saturación de oxígeno basal del grupo N + OCD es 95\% y en el STAF + OCD es del $95 \%$ y sus presiones parciales de oxígeno son normales; estas condiciones no justifican el uso de OCD.

Los resultados mostraron aumento de la PSAP con $8.24 \mathrm{~mm} \mathrm{Hg}$ en el $\mathrm{N}+\mathrm{OCD}$, y $2.84 \mathrm{~mm} \mathrm{Hg}$ en el $\mathrm{N}$. En el grupo retrospectivo no existe una diferencia significativa entre los pacientes tratados con OCD y los tratados sin OCD.
El punto principal, aunque no se aborda en las conclusiones es el que no existen diferencias significativas entre los pacientes tratados con N + OCD y los STAF + OCD, ya que este último constituye su grupo control con el cual se deben establecer las comparaciones. También nos muestra como el grupo N presenta una menor elevación de la PSAP con significancia estadística comparación con el STAF.

\section{Conclusiones y recomendaciones para la práctica}

Los autores se enfocan en comparar el grupo N + OCD y el N, resulta muy evidente el aumento de la PSAP en el primer grupo sin embargo esta comparación es incorrecta ya que se tratan de dos grupos diferentes en sus características; en el primero es mayor la presencia de enfisema y su DLCO\% esta disminuida, esperando un deterioro más acelerado de la CVF y un aumento de la PSAP. Pero al comparar el N + OCD con su verdadero control qué es STAF + OCD encontramos que no existe una diferencia significativa entre ambos, por lo tanto, no podemos concluir que la elevación de la PSAP sea secundaria en efecto de Nitedanib sobre el VEGF.

Este estudio no puede comprobar la hipótesis sobre el aumento de la de la HP en los pacientes tratados con Nitedanib. Y está diferenciación qué describen los autores en sus conclusión, sobre que el efecto de Nitedanib en pacientes con OCD aumenta la HP es incorrecta, ya que igualmente hay un aumento de la PSAP en el grupo que no recibe antifibroticos.

Los resultados muestran una posible limitación de la progresión de la HP en el grupo tratado con Nitedanib con buena clase funcional y sin necesidad de OCD.

Sin embargo con una muestra tan pequeña, con la cantidad de pacientes eliminados durante el estudio que superan el 50\%, con lo heterogéneo del grupo y las múltiples variables confusoras, no es posible mediante este estudio establecer conclusiones firmes acerca del efecto del Nitedanib en la HP.

Los resultados no permiten darnos pautas claras del uso o no de la terapéutica con Nitedanib, sin embargo la hipótesis de origen es sumamente interesante y nos compromete a la observación de la variación de la HP en estos pacientes. Se requieren estudios con mayor población, prospectivos, para establecer conclusiones reales.

\section{Disclosure Statement}

Por el presente el autor declara que no tiene conflictos de interés con respecto a esta transferencia de conocimiento.

Correspondencia: Dr. Marco Hugo Sánchez-Bustillos, Hospital Star Médica, Perif. de la Juventud 6103, Saucito, 31110 Chihuahua, Chih., México, marcohugos@yahoo.com 